\title{
High-Flow Nasal Cannula in a Mixed Adult ICU
}

\author{
Kristina A Gaunt MD, Sarah K Spilman MA, Meghan E Halub MD, Julie A Jackson RRT-ACCS, \\ Keith D Lamb RRT-ACCS, and Sheryl M Sahr MD MSc
}

\begin{abstract}
BACKGROUND: Humidified, high-flow nasal cannula (HFNC) enables mucociliary clearance, accurate oxygen measurement, precise control of flow, and low-level positive airway pressure. There is sparse information concerning the timing of HFNC on patient outcomes such as incidence of adverse events during hospitalization, ICU stay, and post-ICU stay. METHODS: This is a retrospective analysis of a heterogeneous population of medical and trauma ICU patients who received HFNC therapy in a critical care setting. The study sample included 145 subjects who were admitted to the ICU and received HFNC therapy between March 2012 and February 2014. HFNC was delivered by the Fisher \& Paykel Optiflow system. RESULTS: Of the 145 subjects who received HFNC, $35(24.1 \%)$ received mechanical ventilation before HFNC, $21(14.5 \%)$ received mechanical ventilation after $\mathrm{HFNC}$, and $89(61.3 \%)$ never received mechanical ventilation. Delay to first HFNC was moderately associated with unplanned ICU admission and was strongly correlated with the development of ventilator-associated pneumonia. Subjects with a greater length of time between ICU admission and first use of HFNC experienced significantly longer stays in the ICU and post-ICU periods, even after controlling for adverse events and mechanical ventilation. CONCLUSIONS: Study results provide preliminary evidence that early use of HFNC is beneficial in a medical and trauma ICU population, as it was associated with decreased ICU and post-ICU lengths of stay and reduced incidence of adverse events. This suggests that HFNC should be considered early in the ICU as first-line oxygen therapy. Key words: high-flow nasal cannula; Optiflow; intensive care unit; mucociliary clearance; positive airway pressure; mechanical ventilation. [Respir Care 2015;60(10):1383-1389. ( C 2015 Daedalus Enterprises]
\end{abstract}

\section{Introduction}

Many studies regarding the efficacy of humidified highflow nasal cannula (HFNC) have been published in the last decade, and it is recognized that HFNC facilitates muco-

\footnotetext{
Drs Gaunt and Halub are affiliated with the General Surgery Residency Program, Iowa Methodist Medical Center; Ms Spilman and Dr Sahr are affiliated with the Department of Trauma Services, UnityPoint Health; and Ms Jackson and Mr Lamb are affiliated with the Department of Respiratory Therapy, UnityPoint Health, Des Moines, Iowa.
}

Dr Gaunt presented results of this study at the Iowa Committee on Trauma Resident Paper Competition, held October 16, 2014 in Des Moines, Iowa.

Mr Lamb has disclosed relationships with General Electric, Masimo, Bayer, and Sunovian. The other authors have disclosed no conflicts of interest.

Correspondence: Sarah K Spilman MA, Trauma Services, Iowa Methodist Medical Center, 1200 Pleasant Street, Des Moines, IA 50309. E-mail: sarah.spilman@unitypoint.org.

DOI: $10.4187 /$ respcare.04016 ciliary clearance, accurate oxygen flow, and low-level positive airway pressure. ${ }^{1-12}$ HFNC has been used successfully with medical ICU patients, as well as in postoperative cardiac and vascular populations..$^{3,6,10-15}$ One study in the trauma literature supported use of early noninvasive ventilation (NIV) in blunt trauma, ${ }^{16}$ but much work is yet to be done regarding use of HFNC in trauma populations.

Although many studies have focused on the physiological mechanisms and processes of the therapy, 2,5,7-10,14 very few have explored the timing of initiation, relevance to heterogeneous populations, and general patient outcomes. With the benefits of HFNC demonstrated in certain study populations, it is reasonable to explore other aspects and outcomes of the therapy. For example, one meta-analysis suggested that NIV led to a significant reduction in intubation rates, as well as reduced ICU stay, for subjects experiencing acute respiratory failure after chest trauma. ${ }^{17}$ Another review reported that blunt chest trauma subjects benefited from early initiation of NIV, leading to decreased ICU stay, complications, and mortality. ${ }^{16}$ Nevertheless, neither of these studies included HFNC, even though some 
literature has proposed that HFNC is equivalent or superior to standard NIV. ${ }^{4,12}$ If HFNC performs similarly to, or even better than, standard NIV devices, HFNC therapy may have analogous benefits to medical and trauma patients who were found in the aforementioned studies using NIV.

See the Related Editorial on Page 1522

\section{Study Rationale}

There is a paucity of information regarding use of HFNC across heterogeneous populations. In this retrospective analysis of subjects admitted to the ICU, we analyzed the demographic and clinical predictors of HFNC success across a heterogeneous population of ICU subjects. We hypothesized that early use of HFNC would reduce the incidence of adverse events or complications, and ICU and post-ICU lengths of stay.

\section{Methods}

\section{Study Design and Patient Sample}

A retrospective study was conducted at a tertiary hospital with a 24-bed adult ICU (Iowa Methodist Medical Center, Des Moines, Iowa). Subjects were identified prospectively through a respiratory therapy database that tracked daily bedside therapies and therapist assignments. Patients were included in the study if they were age $18 \mathrm{y}$ or older and received HFNC therapy during the study period of March 2012 through February 2014. Decision to initiate HFNC was made by the respiratory therapist in conjunction with a critical care attending physician when supplemental oxygen-delivery devices were ineffective. The population was an equal mix of trauma and medical subjects, which accurately reflects the heterogeneous composition of the ICU at the study hospital.

HFNC was delivered by the Fisher \& Paykel Optiflow system (Fisher \& Paykel Healthcare, Auckland, New Zealand). The system consists of an air-oxygen blender (model 3500HL, Sechrist Industries, Anaheim, California) with adjustable $\mathrm{F}_{\mathrm{IO}_{2}}(21-100 \%)$ that delivers a modifiable gas flow (up to $80 \mathrm{~L} / \mathrm{min}$ ) to a humidification system (Fisher \& Paykel MR 850) where the gas is heated. The gas mixture is then routed through a high performance circuit containing water to be delivered at $37^{\circ} \mathrm{C}$ via short, widebore bi-nasal prongs (RT033 or RT044). At the study hospital, initial settings are routinely fixed at $50 \mathrm{~L} / \mathrm{min}$ and $50 \% \mathrm{~F}_{\mathrm{IO}_{2}}$. No funding or support was received from the manufacturer to conduct this study.

Chart review of the electronic medical record was conducted for data not included in the respiratory therapy

\section{QUICK LOOK}

\section{Current knowledge}

Heated and humidified $\mathrm{O}_{2}$ delivered by high-flow nasal cannula (HFNC) reduces ventilatory requirements by flushing the anatomical dead space and improves oxygenation by meeting inspiratory flow demands. A low level of end-expiratory pressure may also be generated further enhancing oxygenation. Heat and humidity allows high flows to be tolerated and improves patient comfort.

\section{What this paper contributes to our knowledge}

In this retrospective review of heated and humidified HFNC use in a mixed medical surgical population, early use was associated with reduced ICU stay when compared with later implementation. No significant adverse events were identified during HFNC use.

database. Three surgical residents and one critical care physician reviewed the records for data extraction. Interrater reliability was assessed for $10 \%$ of the records to ensure consistency in abstraction. The study was approved by the institutional review board at the hospital. The requirement of subject consent was waived because chart review occurred retrospectively after patient discharge.

\section{Study Variables}

Demographic variables included subject gender, age, body mass index (BMI), residence at home before admission, and discharge disposition (home or facility). Mortality included subjects who died in the hospital or were discharged to hospice. The palliative care variable indicated whether a subject received a palliative care consultation during the stay or was discharged to a hospice setting.

Admitting diagnoses and comorbid health conditions were derived from several sources in the electronic medical record, including admission notes, consultation notes, and discharge summaries. Two broad categories of diagnoses were created: medical diagnoses (including bronchitis, cancer, congestive heart failure, hypercarbia, pleural effusion, pneumonia, and pulmonary edema) and thoracic trauma diagnoses (flail chest, hemothorax, pneumothorax, pulmonary contusion, and rib fractures). Comorbid health conditions included past medical history of asthma, atrial fibrillation, COPD, congestive heart failure, obstructive sleep apnea, and current tobacco use.

For each HFNC session, we recorded several data points from the beginning and end of each session: date and time, $\mathrm{L} / \mathrm{min}, \mathrm{F}_{\mathrm{IO}_{2}}$, heart rate, breathing frequency, $\mathrm{S}_{\mathrm{pO}_{2}}$, 


\section{HFNC IN Adult ICU SubJeCtS}

systolic blood pressure, and diastolic blood pressure. We computed the length of time between ICU admission and initiation of the HFNC; the variable is reported in days to aid in interpretation of results. We also determined whether HFNC therapy was used before or after the subject received mechanical ventilation.

We were interested in whether early use of HFNC was associated with improved patient outcomes. The first outcome of interest was the occurrence of an adverse event or complication. Ventilator-associated pneumonia (VAP) was derived from definitions from the Centers for Disease Control and Prevention, per hospital protocol. An unplanned ICU admission was defined as a return to the ICU after transitioning to a general in-patient floor. An unplanned intubation was indicated if the subject was extubated from mechanical ventilation and subsequently reintubated, or if the subject was intubated for the first time after HFNC. It should be noted that we excluded subjects who received mechanical ventilation only for surgical ventilation as a means of general anesthesia rather than a therapy to treat respiratory insufficiency, and if the duration of mechanical ventilation was $>24 \mathrm{~h}$. Finally, a medical emergency team call was indicated if the alert was activated for the subject on a general in-patient floor.

The second outcome of interest was the ICU stay. ICU stay was calculated such that any partial day counted as a full day. For example, 2 days and 1 night in the ICU were calculated as 2 days in the ICU. A third outcome of interest was the post-ICU stay, which was calculated as the time between the first ICU discharge and the hospital discharge. Subjects were excluded from this outcome if they were discharged from the hospital directly from the ICU.

\section{Statistical Procedures}

All analyses were performed with SPSS Basic Statistics 20.0 (IBM, Armonk, New York). Descriptive statistics were examined and reported for categorical data as counts and percentages, and for continuous data as medians and interquartile ranges (IQR). Correlations between subject characteristics and outcome variables were calculated with Spearman's rho coefficient $\left(\mathrm{r}_{\mathrm{s}}\right)$. Multivariate regression was conducted with saturated main effects models in the first step; variables contributing least to the model were omitted, and only the final equations are presented. All statistical tests were 2-tailed and based on a 0.05 significance level.

\section{Results}

The study sample included 145 subjects; demographic and clinical characteristics of study subjects are summarized in Table 1. Fifty-nine percent of the subjects were male, and the median age was 66 y (IQR: 56-78). The
Table 1. Demographic and Clinical Characteristics of Subjects in the Study

\begin{tabular}{lc}
\hline \hline \multicolumn{1}{c}{ Characteristics } & All Subjects* \\
\hline Male, $n(\%)$ & $85(58.6)$ \\
Age, median (IQR) & $66(56-78)$ \\
Body mass index, median (IQR) & $29.4(24.4-34.2)$ \\
Private home before admission, $n(\%)$ & $132(91.0)$ \\
Trauma patient, $n(\%)$ & $73(50.3)$ \\
Palliative care, $n(\%)$ & $34(23.4)$ \\
Received mechanical ventilation, $n(\%)$ & $56(38.6)$ \\
Hospital days, median (IQR) & $13(9-22)$ \\
ICU days of initial stay, median (IQR) & $6(3-11)$ \\
Discharged home, $n(\%) \dagger$ & $47(40.5)$ \\
Died or discharged to hospice, $n(\%)$ & $29(20.0)$ \\
Past medical history, $n(\%)$ & \\
COPD & $54(37.2)$ \\
Atrial fibrillation & $35(24.1)$ \\
Current smoker & $31(21.4)$ \\
Congestive heart failure & $25(17.2)$ \\
Obstructive sleep apnea & $21(14.5)$ \\
Asthma & $19(13.1)$ \\
\hline$* N=145$. & \\
$\dagger$ Excludes patients who died or were discharged to hospice. & \\
IQR = interquartile range & \\
\hline
\end{tabular}

majority of subjects resided at home before hospital admission, but $41 \%$ of subjects were discharged home after the stay. One-quarter of subjects received palliative care, and $20 \%$ of subjects died in the hospital or were discharged to hospice. A considerable number of subjects had at least one comorbid health condition, with the most frequent conditions including COPD (37.2\%), atrial fibrillation $(24.1 \%)$, and current smoker (21.4\%).

Table 2 shows the admitting diagnoses and complications of subjects in the study. Nearly $60 \%$ of subjects in the study had a medical diagnosis, and $40 \%$ had a thoracic trauma diagnosis. The most common medical diagnoses were pleural effusion, hypercarbia, and pneumonia. Additionally, more than half of subjects were hypoxic, and nearly one-third were tachycardic at the time of admission to the hospital. The most common thoracic trauma diagnoses were rib fractures, pneumothorax, and pulmonary contusion.

Forty-three percent of all subjects experienced at least one adverse event during their stay (see Table 2). More than $20 \%$ of subjects received a medical emergency team call or experienced an unplanned ICU admission, and nearly $20 \%$ of subjects had an unplanned intubation. Seven percent of subjects developed VAP.

Table 3 shows the device settings and patient vital signs from the beginning and end of the initial HFNC therapy. At the start of the therapy, median air flow was $50 \mathrm{~L} / \mathrm{min}$ (IQR: 50-60) and median $\mathrm{F}_{\mathrm{IO}_{2}}$ was $0.50(0.40-0.60)$; there was a 


\section{HFNC IN Adult ICU SubJeCTS}

statistically significant decline in support levels over the course of the therapy. After the HFNC therapy, subjects had significant reductions in heart rate and breathing frequency, but there were no significant differences in $\mathrm{S}_{\mathrm{pO}_{2}}$ or blood pressure. The median time to first HFNC was 1 hospital day, and the initial therapy was approximately $26 \mathrm{~h}$ in duration.

Table 2. Diagnoses and Complications of Subjects in the Study

\begin{tabular}{lc}
\hline \hline \multicolumn{1}{c}{ Medical Diagnoses } & All Subjects* \\
\hline All medical diagnoses, $n(\%)$ & $84(57.9)$ \\
Bronchitis & $18(12.4)$ \\
Cancer & $23(15.9)$ \\
Congestive heart failure & $19(13.1)$ \\
Hypercarbia & $36(24.8)$ \\
Pleural effusion & $39(26.9)$ \\
Pneumonia & $30(20.7)$ \\
Pulmonary edema & $16(11.0)$ \\
Thoracic trauma diagnoses, $n(\%)$ & $57(39.3)$ \\
Flail chest & $7(4.8)$ \\
Hemothorax & $11(7.6)$ \\
Pneumothorax & $29(20.0)$ \\
Pulmonary contusion & $24(16.6)$ \\
Rib fracture(s) & $54(37.2)$ \\
Other admitting conditions, $n(\%)$ & \\
Head injury/bleed & $23(15.9)$ \\
Hypoxia & $78(53.8)$ \\
Tachycardia & $46(31.7)$ \\
Adverse events, $n(\%)$ & $63(43.4)$ \\
Medical emergency team call & $31(21.4)$ \\
Unplanned ICU admission & $31(21.4)$ \\
Unplanned intubation & $28(19.3)$ \\
Ventilator-associated pneumonia & $10(6.9)$ \\
$* N=145$. & \\
\hline
\end{tabular}

\section{Timing of HFNC}

Figure 1 illustrates the timing of mechanical ventilation and HFNC therapy for all subjects in the study. Approximately $25 \%$ of subjects $(n=35)$ received mechanical ventilation before HFNC, and 7 of those subjects (20.0\%) were extubated from mechanical ventilation and subsequently reintubated. The majority of subjects who received mechanical ventilation before HFNC were discharged alive $(n=31 ; 88.6 \%)$, and 6 subjects $(17.1 \%)$ ultimately received a tracheostomy. Of the 110 subjects who did not receive mechanical ventilation before HFNC, 21 (19.1\%) deteriorated on HFNC and were intubated and 5 subjects $(4.5 \%)$ ultimately received a tracheostomy. Nearly $25 \%$ of subjects $(n=25)$ in this group died or were discharged to hospice.

Receiving mechanical ventilation before HFNC was moderately correlated with an admitting diagnosis of hypercarbia $\left(\mathrm{r}_{\mathrm{s}}=0.26, P=.004\right)$, as well as the development of VAP $\left(\mathrm{r}_{\mathrm{s}}=0.29, P<.001\right)$, increased ICU stay $\left(\mathrm{r}_{\mathrm{s}}=0.35, P<.001\right)$, and increased post-ICU stay $\left(\mathrm{r}_{\mathrm{s}}=0.26, P=.002\right)$. Subjects who received mechanical ventilation before $\mathrm{HFNC}$ received their first $\mathrm{HFNC}$ therapy on day 5 of their hospital stay (median $=122 \mathrm{~h}$; IQR: 60-248). In comparison, subjects who did not receive mechanical ventilation before HFNC received their first HFNC therapy $1.5 \mathrm{~d}$ after hospital admission (median $=37 \mathrm{~h}$; IQR: 9-92).

\section{Adverse Events}

Days to first HFNC were not correlated with receiving a medical emergency team call later in the stay and were weakly correlated with unplanned intubation. However, days to first HFNC were moderately correlated with an

Table 3. Device Settings and Subject Vital Signs From the Beginning and End of Initial HFNC Therapy

\begin{tabular}{|c|c|c|c|}
\hline & Start of Initial HFNC Therapy & End of Initial HFNC Therapy & $P$ \\
\hline \multicolumn{4}{|l|}{ HFNC, median (IQR) } \\
\hline $\mathrm{L} / \mathrm{min}$ & $50(50-60)$ & $50(45-60)$ & $<.001$ \\
\hline $\mathrm{F}_{\mathrm{IO}_{2}}$ & $0.50(0.40-0.60)$ & $0.45(0.40-0.55)$ & $<.001$ \\
\hline Heart rate, median beats/min (IQR) & $96(80-108)$ & $89(78-101)$ & $<.001$ \\
\hline Breathing frequency, median breaths/min (IQR) & $20(16-27)$ & $19(15-24)$ & .001 \\
\hline $\mathrm{S}_{\mathrm{pO}_{2}}$, median (IQR) & $95(92-97)$ & $96(93-98)$ & .13 \\
\hline Systolic blood pressure, median mm Hg (IQR) & $126(109-143)$ & $122(111-143)$ & .36 \\
\hline Diastolic blood pressure, median mm Hg (IQR) & $73(63-83)$ & $71(63-84)$ & .25 \\
\hline Days to first HFNC, median (IQR) & $1(0-3)$ & NA & \\
\hline Duration of first HFNC, median h (IQR) & $26: 40(11: 54,50: 51)$ & NA & \\
\hline \multicolumn{4}{|l|}{$\begin{array}{l}N=145 \\
\text { HFNC = high-flow nasal cannula } \\
\text { IQR = interquartile range } \\
\text { NA = not applicable }\end{array}$} \\
\hline
\end{tabular}




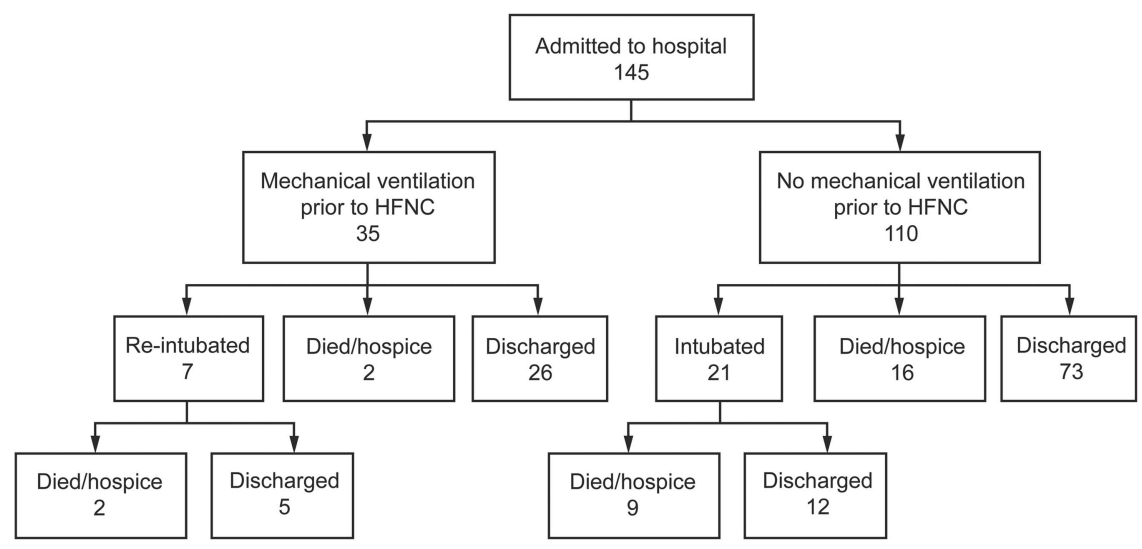

Fig. 1. Flow chart.

Table 4. Regression on ICU Stay

\begin{tabular}{lcc}
\hline \hline & $\beta$ & $P$ \\
\hline Unplanned intubation & 6.17 & $<.001$ \\
Ventilator-associated pneumonia & 8.59 & $<.001$ \\
Mechanical ventilation & 2.36 & .06 \\
Days to first HFNC therapy & 0.47 & $<.001$ \\
Constant & 4.60 & $<.001$ \\
$\mathrm{~F}$ & 43.32 & $<.001$ \\
$\mathrm{R}^{2}$ & 0.55 & $\mathrm{NA}$ \\
& & \\
$N=145$ & & \\
$\mathrm{HFNC}=$ high-flow nasal cannula & & \\
$\mathrm{NA}=$ not applicable & & \\
\hline
\end{tabular}

unplanned ICU admission $\left(\mathrm{r}_{\mathrm{s}}=0.25, P=.003\right)$ and were strongly correlated with development of VAP $\left(\mathrm{r}_{\mathrm{s}}=0.40\right.$, $P<.001)$. Incidence of at least one adverse event was moderately associated with ICU stay $\left(\mathrm{r}_{\mathrm{s}}=0.31, P<.001\right)$ and post-ICU stay $\left(\mathrm{r}_{\mathrm{s}}=0.33, P<.001\right)$.

\section{ICU Stay}

Regression coefficients associated with ICU stay in the final multivariable model are presented in Table 4. Among a heterogeneous population of ICU patients, the incidence of mechanical ventilation at any time did not significantly predict ICU stay. Analyses revealed, however, that having an unplanned intubation during hospitalization and developing VAP were highly predictive of ICU stay. Additionally, the number of days between ICU admission and first HFNC significantly increased ICU stay, even after controlling for mechanical ventilation and adverse events.

\section{Post-ICU Stay}

Multiple regression analyses were conducted to examine the relationship between predictor variables and post-
Table 5. Regression on Post-ICU Stay for Subjects Discharged From the ICU

\begin{tabular}{lcc}
\hline \hline & $\beta$ & $P$ \\
\hline ICU stay & 0.78 & $<.001$ \\
Unplanned ICU admission & 7.67 & .01 \\
Mechanical ventilation & 0.98 & .73 \\
Days to first HFNC therapy & 0.80 & .003 \\
Constant & 7.94 & .009 \\
$\mathrm{~F}$ & 18.89 & $<.001$ \\
$\mathrm{R}^{2}$ & 0.38 & $\mathrm{NA}$ \\
& & \\
A total of 129 subjects were discharged. & & \\
HFNC = high-flow nasal cannula & & \\
NA = not applicable & & \\
\hline
\end{tabular}

ICU stay, and results are shown in Table 5. Receiving mechanical ventilation did not have a significant effect on post-ICU stay. However, length of time in the ICU and unplanned ICU admission were each associated with an increased post-ICU stay. Additionally, days to first HFNC therapy were associated with an increase in post-ICU stay.

\section{Discussion}

The purpose of this study was to provide information regarding demographic and clinical predictors of HFNC success across a heterogeneous population of medical and trauma patients. We found that when HFNC was started earlier in the hospital stay, subjects experienced shorter lengths of stay in the ICU and in the post-ICU periods, as well as fewer adverse events. These relationships remained significant even after controlling for other important events that could lengthen hospitalization, such as mechanical ventilation and unplanned intubation.

As mentioned previously, a number of prior studies have shown the beneficial use of HFNC ${ }^{1-12}$; however, ICU and post-ICU lengths of stay have rarely been studied as out- 


\section{HFNC IN Adult ICU SubJeCtS}

come variables. The results of our study indicate that HFNC, when instituted early in the ICU course, is associated with decreased length of ICU stay and reduced post-ICU stay. Because our study population was heterogeneous in nature, results suggest that a broad range of ICU patients can benefit from early use of HFNC therapy. Further investigation is warranted to determine the efficacy of early, first-line use of HFNC upon admission to the critical care setting, or even as early as in the emergency department before ICU admission.

It is notable that the effect of the timing of HFNC remained highly significant in the regression equations, even after controlling for other confounders. For example, unplanned intubation and delay to HFNC are intuitively related but only weakly correlated $\left(\mathrm{r}_{\mathrm{s}}=0.17, P=.05\right)$, yet both variables were significant predictors of ICU stay. Results demonstrate that, when controlling for other important events that occur during hospitalization, every 1-day delay to HFNC increases ICU stay by one-half day. This suggests that first-line HFNC therapy may play a significant role in reducing ICU and hospital stay.

A recent study by Messika and colleagues ${ }^{12}$ found that subjects who tolerated HFNC had sustained benefits when they did not require intubation. In our analysis, there was a $20 \%$ intubation rate in subjects initially treated with HFNC. However, we were unable to identify significant factors predicting failure of HFNC. Hypercarbia, pleural effusions, and spinal injury were weakly but significantly correlated with unplanned intubation. Arguably, these are indicators of poorly controlled medical illness or sudden decompensation in medical status. Further studies should be performed in these particular patient populations that may elucidate how early use of HFNC can reduce intubation rates.

It should also be noted that $20 \%$ of our study population died or were discharged to hospice. There were 34 palliative care subjects in the study: $5(14.7 \%)$ received mechanical ventilation before HFNC, 8 (23.5\%) received mechanical ventilation after HFNC, and 21 (61.8\%) never received mechanical ventilation. Thus, many of the palliative care subjects may have experienced HFNC as a means of palliation, as the majority did not have escalation of therapy to mechanical ventilation. For subjects with acute respiratory failure, multiple comorbidities, and do-not-intubate orders, HFNC may be a useful means of palliation. Peters and colleagues ${ }^{18}$ reported that HFNC provided adequate oxygenation for $82 \%$ of subjects with do-not-intubate orders without requiring escalation to NIV. To our knowledge, the use of HFNC has not been studied in a palliative population, and further examination is warranted to determine its therapeutic utility.

Lastly, it deserves mention that HFNC therapy can only be administered in the ICU at the study hospital. It is possible that some subjects did not warrant ICU admission except for the requisite HFNC therapy. Plans are under way to expand HFNC therapy to the floor, which could further decrease overall stay, reduce the likelihood of patients experiencing adverse events, and ease the costs associated with critical care hospitalization.

\section{Limitations}

This study has several limitations. The first limitation is that data were collected retrospectively, and we could only ascertain information that was documented in the electronic medical record. Second, because the subject population is a heterogeneous mix of medical and trauma patients, it includes a mixture of medical conditions. Therefore, generalizations cannot be made about any specific subsample of diagnoses. Third, we do not have a control sample of subjects who were similar in acuity but did not receive HFNC, so determinations about the success of the therapy are made with caution.

\section{Conclusions}

This study of heterogeneous populations using HFNC shows that there may be a benefit to using HFNC earlier in the hospital course as a means to reduce the incidence of adverse events and decrease ICU and post-ICU hospital stays.

\section{ACKNOWLEDGMENTS}

We thank the following individuals for their assistance on data collection and analysis: H Clay Dean (Iowa Methodist Medical Center), Trevor W Oetting (UnityPoint Health), and Catherine Hackett Renner (UnityPoint Health).

\section{REFERENCES}

1. Branson RD, Gentile MA. Is humidification always necessary during noninvasive ventilation in the hospital? Respir Care 2010;55(2):209216; discussion: 216.

2. Dysart K, Miller TL, Wolfson MR, Shaffer TH. Research in high flow therapy: mechanisms of action. Respir Med 2009;103(10):1400-1405.

3. Kernick J, Magarey J. What is the evidence for the use of high flow nasal cannula oxygen in adult patients admitted to critical care units? A systematic review. Aust Crit Care 2010;23(2):53-70.

4. Lee JH, Rehder KJ, Williford L, Cheifetz IM, Turner DA. Use of high flow nasal cannula in critically ill infants, children, and adults: a critical review of the literature. Intensive Care Med 2013;39(2):247-257.

5. Parke R, McGuinness S, Eccleston M. Nasal high-flow therapy delivers low level positive airway pressure. Br J Anaesth 2009;103(6): 886-890.

6. Parke RL, McGuinness SP, Eccleston ML. A preliminary randomized controlled trial to assess effectiveness of nasal high-flow oxygen in intensive care patients. Respir Care 2011;56(3):265-270.

7. Ritchie JE, Williams AB, Gerard C, Hockey H. Evaluation of a humidified nasal high-flow oxygen system, using oxygraphy, capnography and measurement of upper airway pressures. Anaesth Intensive Care 2011;39(6):1103-1110.

8. Roca O, Riera J, Torres F, Masclans JR. High-flow oxygen therapy in acute respiratory failure. Respir Care 2010;55(4):408-413. 


\section{HFNC in Adult ICU SubJeCtS}

9. Rittayamai N, Tscheikuna J, Rujiwit P. High-flow nasal cannula versus conventional oxygen therapy after endotracheal extubation: a randomized crossover physiologic study. Respir Care 2014;59(4):485-490.

10. Sztrymf B, Messika J, Bertrand F, Hurel D, Leon R, Dreyfuss D, et al. Beneficial effects of humidified high flow nasal oxygen in critical care patients: a prospective pilot study. Intensive Care Med 2011;37(11):1780-1786.

11. Sztrymf B, Messika J, Mayot T, Lenglet H, Dreyfuss D, Ricard JD. Impact of high-flow nasal cannula oxygen therapy on intensive care unit patients with acute respiratory failure: a prospective observational study. J Crit Care 2012;27(3):324,e9-e13.

12. Messika J, Ahmed KB, Gaudry S, Miguel-Montanes R, Rafat C, Sztrymf B, et al. Use of high-flow nasal cannula oxygen therapy in subjects with ARDS: a 1-year observational study. Respir Care 2015; 60(2):162-169.

13. Eccleston ML. A prospective observational study of nasal high flow in a cardiothoracic and vascular intensive care and high dependency unit. Master's thesis, The University of Auckland, 2011.
14. Corley A, Caruana LR, Barnett AG, Tronstad O, Fraser JF. Oxygen delivery through high-flow nasal cannulae increase end-expiratory lung volume and reduce respiratory rate in postcardiac surgical patients. Brit J Anaesth 2011;107(6):9981004.

15. Parke RL, McGuinness SP, Dixon R, Jull A. Protocol for a randomised controlled trial of nasal high flow oxygen therapy compared to standard care in patients following cardiac surgery: the HOT-AS study. Int J Nurs Stud 2012;49(3):338-344.

16. Duggal A, Perez P, Golan E, Tremblay L, Sinuff T. Safety and efficacy of noninvasive ventilation in patients with blunt chest trauma: a systematic review. Crit Care 2013;17(4):R142.

17. Chiumello D, Coppola S, Froio S, Gregoretti C, Consonni D. Noninvasive ventilation in chest trauma: systematic review and metaanalysis. Intensive Care Med 2013;39(7):1171-1180.

18. Peters SG, Holets SR, Gay PC. High-flow nasal cannula therapy in do-not-intubate patients with hypoxemic respiratory distress. Respir Care 2013;58(4):597-600.

This article is approved for Continuing Respiratory Care Education credit. For information and to obtain your CRCE

(free to AARC members) visit www.rcjournal.com 